\title{
Observations of Geomagnetically Trapped Anomalous Cosmic Rays by SAMPEX
}

\author{
R.S. SELESNICK, J.R. CUMMINGS, R.A. MEWALDT \\ California Instilue of Techadogy, Paradena
}

\begin{abstract}
The first detailed measurements of a belt of geomagnetically trapped heavy ions that originated as interplanetary anomalous cosmic rays (ACRs) are being made by the polar orbiting satellite SAMPEX. The singly ionized interplanetary ACRs are trapped after losing electrons in the upper atmosphere. Their subsequent lifetime against energy loss by ionization of the atmosphere allows them to reach a substantially higher intensity than in interplanetary space. The ACR composition, which includes only elements with high first ionization potentials, is reflected in the trapped ACRs with some bias due to the trapping mechanism. The elements $\mathrm{O}, \mathrm{N}$, and $\mathrm{Ne}$ are present, while the lower atomic number elements, $\mathrm{He}$ and $\mathrm{C}$, are either absent or substantially depleted relative to their interplanetary abundances. The trapping mechanism also determines the location of the ACR belt, which is confined to a narrow region near $\mathrm{L}=2$, and the pitch-angle distribution of the trapped ACRs, which is nearly isotropic except for the well-defined loss cones. The intensities of the trapped and interplanetary ACRs have been measured by SAMPEX since its July, 1992 launch. Both have been steadily increasing with the approach of the minimum of the solar sunspot cycle.
\end{abstract}

\section{Introduction}

The possibility that interplanetary anomalous cosmic rays (ACRs) can be trapped in the geomagnetic field was proposed by Blake and Friesen [1977]. Their theory suggested that the singly ionized ACRs, upon reaching the upper atmosphere at mid-latitudes, may be stripped of some or all of their remaining electrons thus undergoing an abrupt increase in magnetic rigidity. The subsequent trajectories are more tightly constrained by the geomagnetic field and, under the right circumstances, they will remain trapped. The first strong observational evidence in support of the theory was inferred from data taken by the Cosmos satellites with passive track detectors [Grigorov et al., 1991]. The composition, angular distribution, and temporal variations of the Cosmos data were consistent with a trapped ACR population, although the track detectors did not provide direct measurements of the ions' spatial distribution. Confirmation that they were in a region of stable geomagnetic trapping came from measurements of $\mathrm{N}, \mathrm{O}$, and $\mathrm{Ne}$ ions at kinetic energies of $\gtrsim 15 \mathrm{MeV} /$ nucleon, with spatial and temporal resolution, by the Mass Spectrometer Telescope (MAST) on the polar orbiting SAMPEX satellite [Cummings et al., 1993, 1994]. Detailed anlysis and interpretation of the SAMPEX data is given by Selesnick et al. [1994] and their results are summarized here.

\section{Trapped Particle Intensities}

The method employed for calculating the trapped particle intensities is described first. Detailed distribution functions are calculated only for oxygen because it is the dominant element in the data. However, energy dependent abundances, relative to oxygen, are provided for carbon, nitrogen, and neon.

\section{Method of calculation}

The intensity at the spacecraft $j(E, \alpha, L, t)$ can, in general, be described as a function of kinetic energy per nucleon $E$, pitch-angle $\alpha$ relative to the local magnetic field, magnetic $L$ shell, and time $t$. Each observed particle is characterized by its measured kinetic energy, nuclear charge, mass, trajectory through the telescope, and the time of observation. From these properties, and the information available about the spacecraft orbit and pointing directions, and the geomagnetic field model, it is straightforward to calculate $E, L$, and $\alpha$ (the IGRF 1985 model with secular variations was used to calculate all magnetic field parameters). The local and equatorial values of $\alpha$ and the magnetic field magnitude $B$ are related by

$$
\frac{\sin ^{2} \alpha}{B}=\frac{\sin ^{2} \alpha_{0}}{B_{0}}
$$


where the subscript 0 denotes equatorial values. Ideally, one would calculate from the local measurements the equatorial intensity $j_{0}$. However, because the spacecraft orbits at low altitude (520 to $675 \mathrm{~km}$ ) there is only a small range of $\alpha_{0}$ near zero (and $180^{\circ}$ ) that is observable. Typical values of $B / B_{0}$ are $\sim 5$ to 6 . It is convenient to introduce a new set of variables $R$ and $\Lambda$, the dipole equivalent radius and magnetic latitude, that are related to $L$ and $B / B_{0}$ by

$$
\begin{gathered}
R=L \cos ^{2} \Lambda \\
\frac{B}{B_{0}}=\frac{L^{3}}{R^{3}}\left(4-\frac{3 R}{L}\right)^{1 / 2}
\end{gathered}
$$

The data are taken at various $R$ and $\Lambda$ values along the SAMPEX orbit. By converting the measured pitchangles to the values that would be observed on the same $L$ shells at a single value of $R=R_{1}$ the pitch-angle distribution is similar to that actually observed and is easily converted to the equivalent distribution at any other point along the field line. The intensity $j_{1}$ at $R=R_{1}$ is related to the local intensity by Liouville's theorem $j_{1}\left(E, \alpha_{1}, L, t\right)=j(E, \alpha, L, t)$. The new pitchangle $\alpha_{1}$ is related to $\alpha$ by

$$
\sin ^{2} \alpha_{1}=\frac{B_{0}}{B} \frac{L^{3}}{R_{1}^{3}}\left(4-\frac{3 R_{1}}{L}\right)^{1 / 2} \sin ^{2} \alpha
$$

In addition, the loss-cone angles at different $R$ values are converted" to nearly constant value of $\alpha_{1}$. We have adopted a value of $R_{1}=1.3$ which is near the upper limit of values reached by the spacecraft in the region where the data where observed, so that only a few $(\sim 5 \%)$ of the observed particles have their mirror points above this value of $R$ and are not included in the calculated intensities.

To calculate $j_{1}\left(E, \alpha_{1}, L, t\right)$ the data are divided into bins. The number of events, $N_{i k n m}$, observed in the $i$ th energy bin from $E_{i}$ to $E_{i+1}$, the $k$ th pitch-angle bin from $\alpha_{1 k}$ to $\alpha_{1 k+1}$, the $n$th $L$ shell bin from $L_{n}$ to $L_{n+1}$, and the $m$ th time bin from $t_{m}$ to $t_{m+1}$ is related to $j_{1}$ by

$$
\begin{gathered}
N_{i k n m}=\int_{0}^{2 \pi} \int_{\alpha_{1 k}}^{\alpha_{1 k+1}} \int_{E_{1}}^{E_{1+1}} \int_{\substack{t_{m} \\
L_{n}<L<L_{n+1}}}^{t_{m+1}} A(E, \theta(\alpha, \beta), \phi(\alpha, \beta)) \\
\cdot \cos \theta(\alpha, \beta) j_{1}\left(E, \alpha_{1}, t, L\right) \sin \alpha \frac{d \alpha}{d \alpha_{1}} d t d E d \alpha_{1} d \beta(4)
\end{gathered}
$$

where: $(\alpha, \beta)$ are spherical angles relative to the local magnetic field, $(\theta, \phi)$ are spherical angles relative to the telescope axis, and $A$ is the telescope response function which has units of area. The integral over $t$ includes only times when the spacecraft $L$, which is a function of $t$, is within the $n$th $L$ shell bin. The simplest way to solve for $j_{1}$ is to assume that within each $i, k, n, m$ interval it is a constant, $j_{i k n m}$. Then

$$
\begin{aligned}
j_{i k n m}= & N_{i k n m}\left[2 \pi \int_{\alpha_{1 k}}^{\alpha_{1 k+1}} \int_{E_{1}}^{E_{1+1}} \int_{L_{n}<L<L_{n+1}}^{t_{m+1}}\right. \\
& \left.H\left(E, \alpha\left(\alpha_{1}, t\right), \theta_{B}(t)\right) \frac{d \alpha}{d \alpha_{1}} d t d E d \alpha_{1}\right]^{-1}
\end{aligned}
$$

where

$$
\begin{aligned}
& H\left(E, \alpha, \theta_{B}\right)=\frac{1}{2 \pi} \\
& \quad \int_{0}^{2 \pi} A(E, \theta(\alpha, \beta), \phi(\alpha, \beta)) \cos \theta(\alpha, \beta) \sin \alpha d \beta
\end{aligned}
$$

is the effective area of the telescope averaged over the gyrophase angle $\beta . H$ is a function of $E, \alpha$, and the angle $\theta_{B}$ between the telescope axis and the magnetic field. It is related to the standard telescope geometry factor, $G$, by

$$
G(E)=2 \pi \int_{0}^{\pi} H\left(E, \alpha, \theta_{B}\right) d \alpha
$$

which is independent of $\theta_{B}$. However, $G$ can only be used when $j$ is isotropic (independent of $\alpha$ ). Note that $H$ is a function of time through $\theta_{B}$, so that the integral of $H$ in (8) takes into account the amount of time spent at each $\theta_{B}$ during the time interval $m$. For example, it is possible that most of the time is spent at a $\theta_{B}$ where $H$ is small, so a small number of counts could still mean a high intensity. Since the spacecraft does not orbit at constant $B / B_{0}$, there is also a time dependence in $\alpha$ when it is translated from $\alpha_{1}$ for evaluation of $H$. This is also taken into account in (8).

To calculate $H$ from (9) we have used a Monte Carlo integration. Particle trajectories were chosen with fixed $\theta_{B}$ and $\alpha$ values, while $\beta$ and the position that they enter the telescope were varied at random. The trajectories were followed through the geometry of MAST until they either stopped, according to a theoretical range-energy relationship, or exited the telescope. Trajectories were included in the integration if they satisfied the same set of selection criteria as was applied to the analysis of the flight data. The values of $\theta_{B}$ and $\alpha$ were then varied in steps of $10^{\circ}$ over the range of possible values. Intermediate values for the integrations in (8) were found by linear interpolation. To test the procedure, we have compared numerical integrations of $H$ 
according to (10) with a direct Monte Carlo calculation of $G$. The results were consistent within the statistical errors of the Monte Carlo integrations for all $\theta_{B}$ values chosen. Some sample plots of $H$ versus $E$ for oxygen at selected $\alpha$ and $\theta_{B}$ are shown in Figure 1. They illustrate the acceptance cone angle of MAST $\left(\sim 50^{\circ}\right)$ and its convolution with the cone of trajectories at a constant pitch-angle $\alpha$.

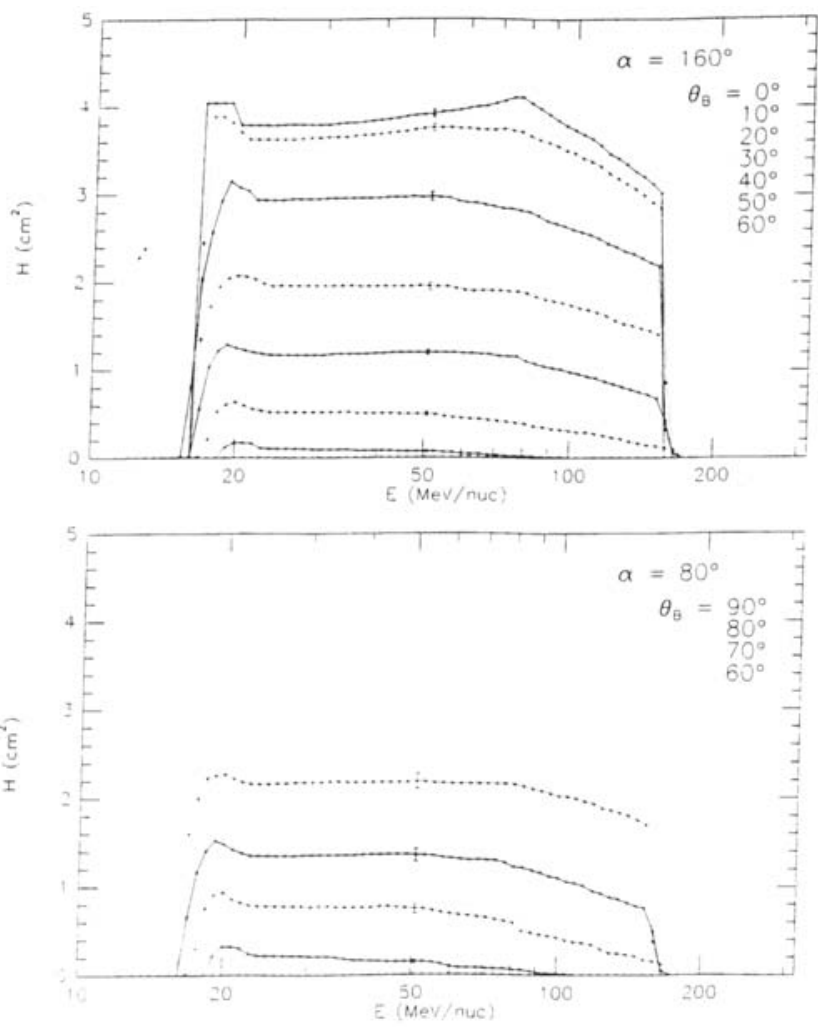

Figure 1. The effective area of MAST averaged over gyro-phase, $H$, as a function of energy for oxygen, at various selected values of the telescope angle relative to $B$ and the particle pitch-angle. Normalization errors due to the Monte Carlo integration are shown near the center of each curve.

It is impractical to use (8) directly to calculate $j_{1}$ because it is a function of four variables and the data are limited so the number of particles in each bin would be small. Instead, we have chosen to represent $j_{1}$ as a separable function

$$
j_{1}\left(E, \alpha_{1}, L, t\right)=U(E, L) V\left(\alpha_{1}\right) W(t)
$$

As shown in the results below, we found it necessary to maintain the $E$ and $L$ dependences in a single function, but separating the functions of $\alpha_{1}$ and $t$ was a reasonable approximation given the limitations of the data. The three functions $U, V$, and $W$ were calculated as follows: Each function was assumed to be constant within each bin, so that, for example, $V_{k}$ represents the value of $V$ within the $k$ th $\alpha_{1}$ bin. Initial values of $V$ and $W$ were chosen to be 1 for all $\alpha_{1}$ and $t$ bins. An iterative solution was then found by successively evaluating

$$
\begin{gathered}
U_{i n}=\frac{\sum_{k, m} N_{i k n m}}{\sum_{k, m} V_{k} W_{m} \bar{H}_{i k n m}} \\
V_{k}=\frac{\sum_{i, n, m} N_{i k n m}}{\sum_{i, n, m} U_{i n} W_{m} \bar{H}_{i k n m}} \\
W_{m}=\frac{\sum_{i, k, n} N_{i k n m}}{\sum_{i, k, n} U_{i n} V_{k} \bar{H}_{i k n m}}
\end{gathered}
$$

where $\bar{H}_{i k n m}$ is the factor in brackets in (8). The sequence (12) was repeatedly evaluated until the values of $U, V$, and $W$ converged. In practice only a few iterations were required.

\section{Results}

The trapped ACRs are observed over a region southeast of the South Atlantic anomaly between the southern tips of South America and Africa [Cummings et al., $1993,1994]$. The intensities of oxygen nuclei observed in this region during a $\sim 20$ month period beginning in July 1992, when SAMPEX was launched, have been evaluated with the method described above. The intensity units were retained in the function $U(E, L)$ which is shown as a function of $E$ and $L$ in Figures 2 and 3 respectively. The functions $V\left(\alpha_{1}\right)$ and $W(t)$ and are shown in Figures 4 and 5 respectively. Due to the normalizations chosen for $V$ and $W, U$ represents the average intensity perpendicular to the magnetic field.

Starting with the energy spectra (the $E$ dependence of $U$ ), these were evaluated in 8 energy bins logarithmically spaced from 15 to $105 \mathrm{MeV} /$ nucleon. Each spectrum was evaluated over a range in $L$ corresponding to a $2^{\circ}$ change in invariant latitude $\left(\cos ^{-1}\left[L^{-1 / 2}\right]\right)$ from $38^{\circ}$ to $50^{\circ}$. The $L$ value at the center of each $L$ bin is shown above each of the six spectra in Figure 2. The spectra show a general trend of flattening at the lower energies as $L$ decreases. This trend is expected because the cutoff rigidity increases with decreasing $L$, prohibiting access for the lower energy particles. The lower energies then fill in due to energy loss of the trapped particles producing a nearly flat spectrum [Blake, 1990]. 


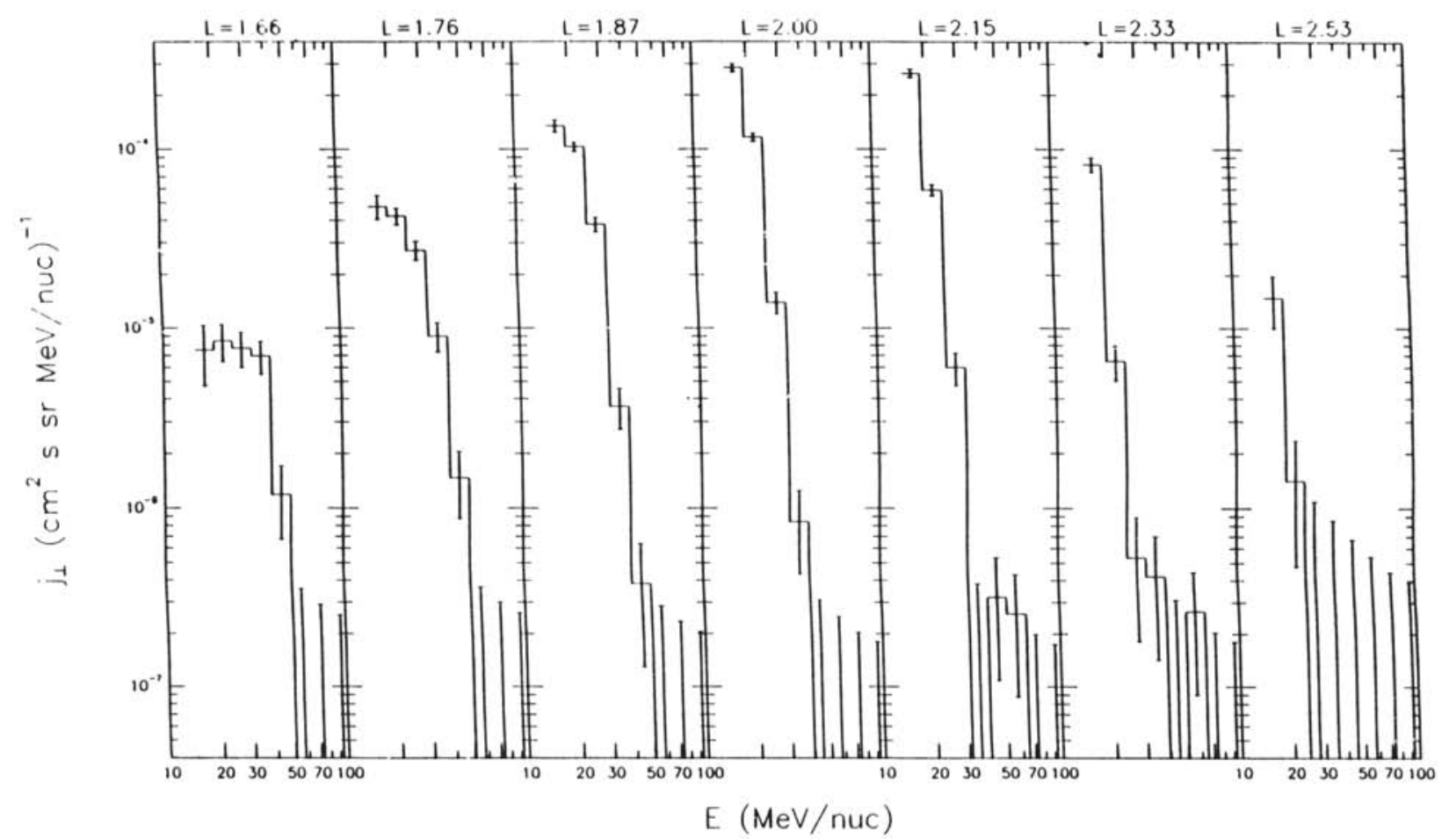

Figure 2. Average energy spe tra of trapped $O$ perpendicular to the magn tic field. The data were divided into bins of $2^{\circ}$ invariant iatitude and the $L$ shell at the center of each bin is shown above each spectrum.

The data from Figure 2 are also shown as a function of $L$ for 4 energy intervals in Figure 3 . The 15 to 19 $\mathrm{MeV} /$ nucleon intensity is seen to be peaked at $L \approx 2.1$, while the peak intensity is at lower $L$ values for higher energies as expected on the basis of cutoff considerations. The total number of events observed in each $L$ bin is also shown in Figure 3.

The pitch-apgle distribution, $V\left(\alpha_{1}\right)$, is shown in the top panel of Figure 4 . It was calculated for $5^{\circ}$ bins in $\alpha_{1}$ and normalized to the average of the values on each side of $90^{\circ}$. Since the observations were in the southern hemisphere, low and high values of $\alpha_{1}$ correspond respectively to upward and downward trajectories. The distribution is nearly isotropic in the region outside the atmospheric loss cones, whose edges are at $\sim 65$ to $70^{\circ}$ and $\sim 110$ to $115^{\circ}$. This would be expected if the interplanetary source were constant over a long time period prior to the observations and if the stripping process occurred in a single atomic interaction. Then both the source and loss rates of the trapped ACRs would be proportional to the atmospheric density at the mirror point altitude, so their ratio, which determines the intensity, would be independent of the mirror point and therefore of the pitch-angle at a given altitude. If the electron stripping occurred in more than one step then the probability of stripping and the source-

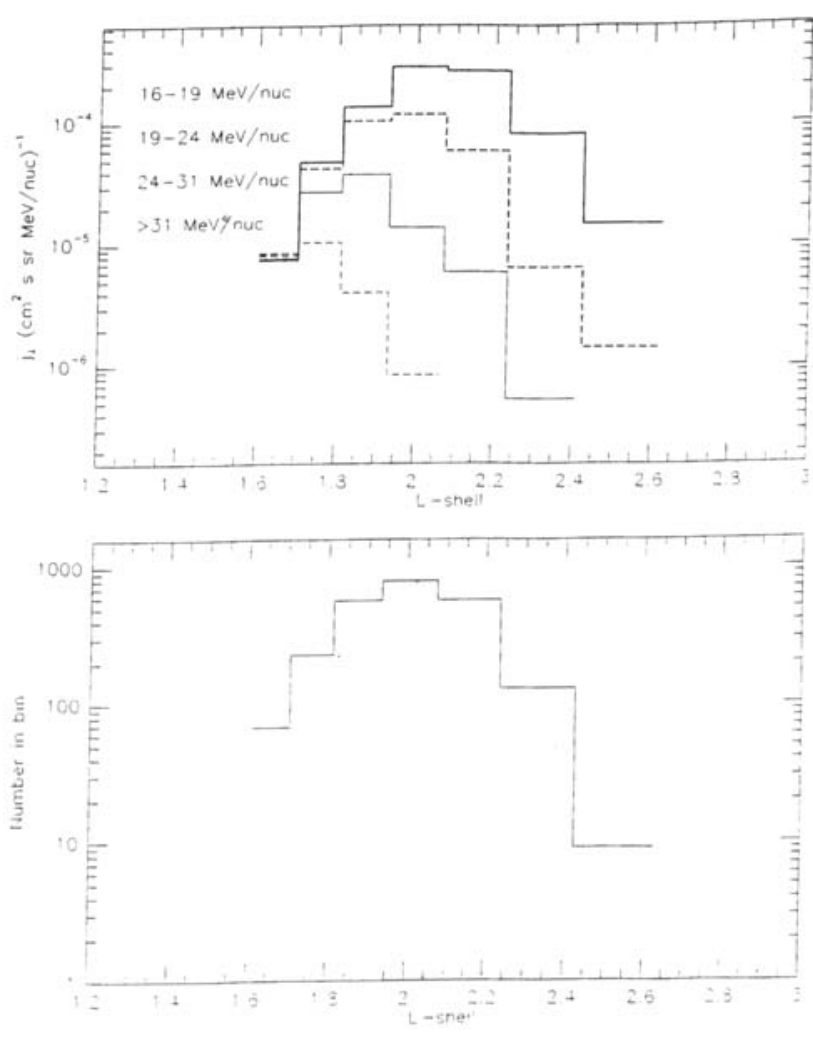

Figure 3. (Top) The average intensity versus $L$ shell of $90^{\circ}$ pitch-angle trapped $\mathrm{O}$ at $R=1.3$ in 4 energy ranges. (Bottom) The total number of $O$ nuclei observed in each $2^{\circ}$ invariant latitude $L$ shell bin. 
strength would be stronger functions of the atmospheric density than the loss rate. The pitch angle distribution would then be peaked near the edges of the loss cone where the mirror points are lowest. The nearly isotropic distribution observed outside the loss cones therefore suggests that the stripping is a single-step process, and this should continue to be true at higher altitudes. However, because the particle lifetimes increase with mirror point altitude, there should perhaps be a local minimum in the pitch-angle distribution at $90^{\circ}$ when the interplanetary intensity is increasing with time. There is in fact some indication of such a local minimum in Figure 4.

The pitch-angle distribution in Figure 4 shows that the intensities inside the loss cones are consistent with much lower values than those outside, although the upper limits at small pitch-angles are relatively high due to the small amount of time that SAMPEX spends pointing down. The intensities in the loss-cones, shown by the dashed lines in Figure 4, were not included in the summations of (12a) and (12c), so that they do not contribute to the total intensity, for the following reasons: They are not part of the trapped distribution,
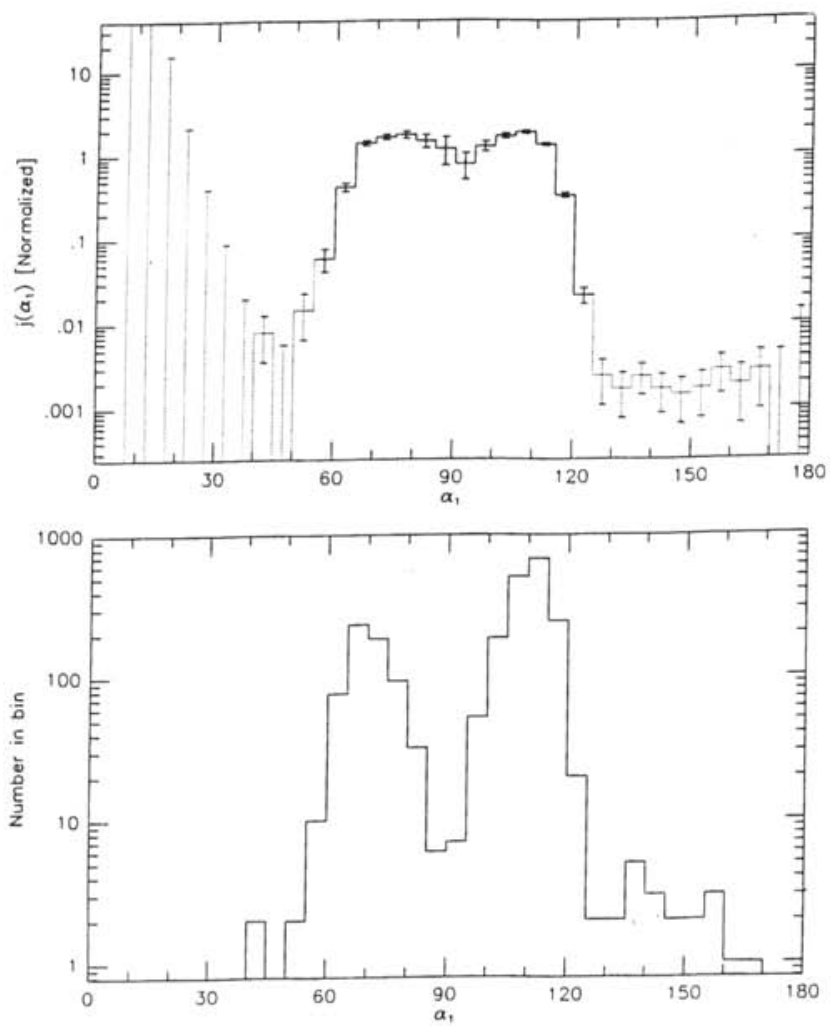

Figure 4. (Top) Normalized pitch-angle distribution of trapped $O$. The pitch-angles were transformed to $R=$ 1.3 as described in the text. (Bottom) The total number of $\mathrm{O}$ nuclei observed in each $5^{\circ}$ pitch-angle bin. and their values are expected to be inaccurate both due to the low number of counts and because a nontrapped distribution will not necessarily be gyrotropic (independent of $\beta$ ) as assumed in the calculation of $H$. The number of counts in each bin are also shown in Figure 4. The double peak is due to the separation of the pitch-angles away from $90^{\circ}$ when they are converted from $\alpha$ to $\alpha_{1}$.

The variation of the trapped intensity with time, $W(t)$, is shown in Figure 5 with a similar format to Figure 4. The bins are 15 days wide and the normalization is to an average value of 1 over the entire period. The number of counts in each bin show a 3 month periodicity due to the precession of the SAMPEX orbit, which provides more favorable viewing geometry when the orbit is in the noon-midnight plane rather than dawn-dusk. That the periodicity is not evident in the intensity versus time demonstrates that the changes in viewing geometry have been properly compensated for, thereby validating the calculation of the pitchangle distribution. The general trend of increasing intensity is a result of the corresponding increase in the interplanetary ACR intensity with the approach to solar
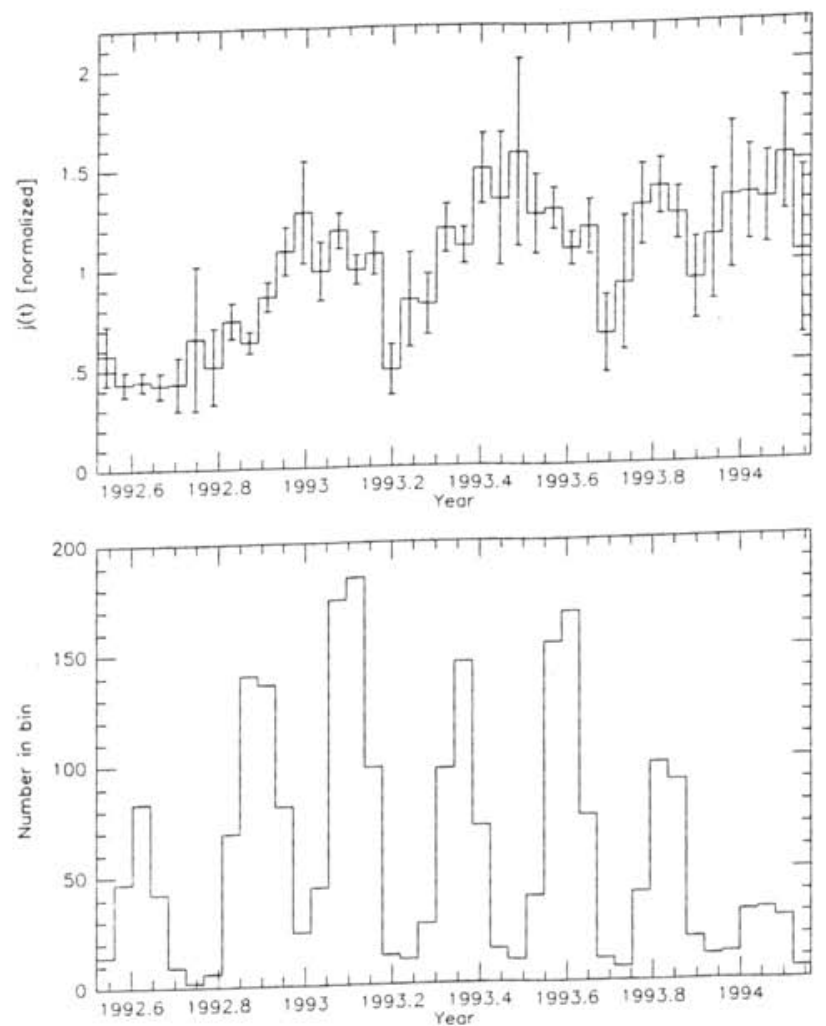

Figure 5. (Top) Normalized intensity of trapped $O$ versus time. (Bottom) The total number of $O$ nuclei observed in each 15 day time bin. 
minimum [Mewaldt et al., 1993]. The decrease in the peak counting rates with time since mid-1993 (bottom panel of Figure 5) is due to an instrumental problem that is corrected for in the data analysis.

To compare the relative abundances of the elements in different energy ranges, integral energy spectra for trapped $\mathrm{C}, \mathrm{N}, \mathrm{O}$, and $\mathrm{Ne}$ are shown in Figure 6. Each point at a given energy represents the relative intensity. of each element for all particles observed above that energy. The data were normalized by the average geometry factors for each energy bin, but the time interval, which is equal for each element and energy bin, was not taken into account. The results in Figure 6 show that, when compared on the basis of energy per nucleon $E$, the relative abundances of trapped $\mathrm{N}, \mathrm{O}$, and $\mathrm{Ne}$ vary with $E$ in such a way that the integral spectra soften with increasing $Z$. The integral energy spectrs shown in Figure 6 are consistent with the $L=2$ and $L=2.15$ spectra in Figure 6 where the intensities are greatest. A typical interplanetary ACR spectrum for the same energy range is also shown in Figure 6 for comparison.

\section{Conclusion}

The MAST instrument on SAMPEX has made the first detailed measurements of the composition, energy spectra, pitch-angle distribution, spatial distribution, and recent time variations of the geomagnetically trapped anomalous cosmic rays. These data have provided strong support for the original theory of Blake and Friesen [Selesnick et al., 1994] and will provide new constraints for more detailed theoretical investigations. SAMPEX and MAST are still operating and continue to return data on these and other particle populations.

Acknowledgments. This work was supported by NASA under contract NAS5-30704 and grant NAGW-1919.

\section{References}

Blake, J. B., and L. M. Friesen, A technique to determine the charge state of the anomalous low energy cosmic rays, Proc. 15th Internat. Cosmic Ray Conf. (Ploudiv), 2, 341-346, 1977

Blake, J. B., Geomagnetically trapped heavy ions from anomalous cosmic rays, Proc. 21st Internat. Cosmic Ray Conf. (Adelaide), 7, 30-33, 1990.

Cook, W. R., A. C. Cummings, J. R. Cummings, T. L. Garrard, B. Kecman, R. A. Mewaldt, R. S. Selesnick, E. C. Stone, and T. T. von Rosenvinge, MAST: A mass spectrometer telescope for studies of the isotopic composition of solar, anomalous, and galactic cosmic ray nuclei, IEEE Trans. Geoscience Remote Sensing, 91, 557-564, 1993.

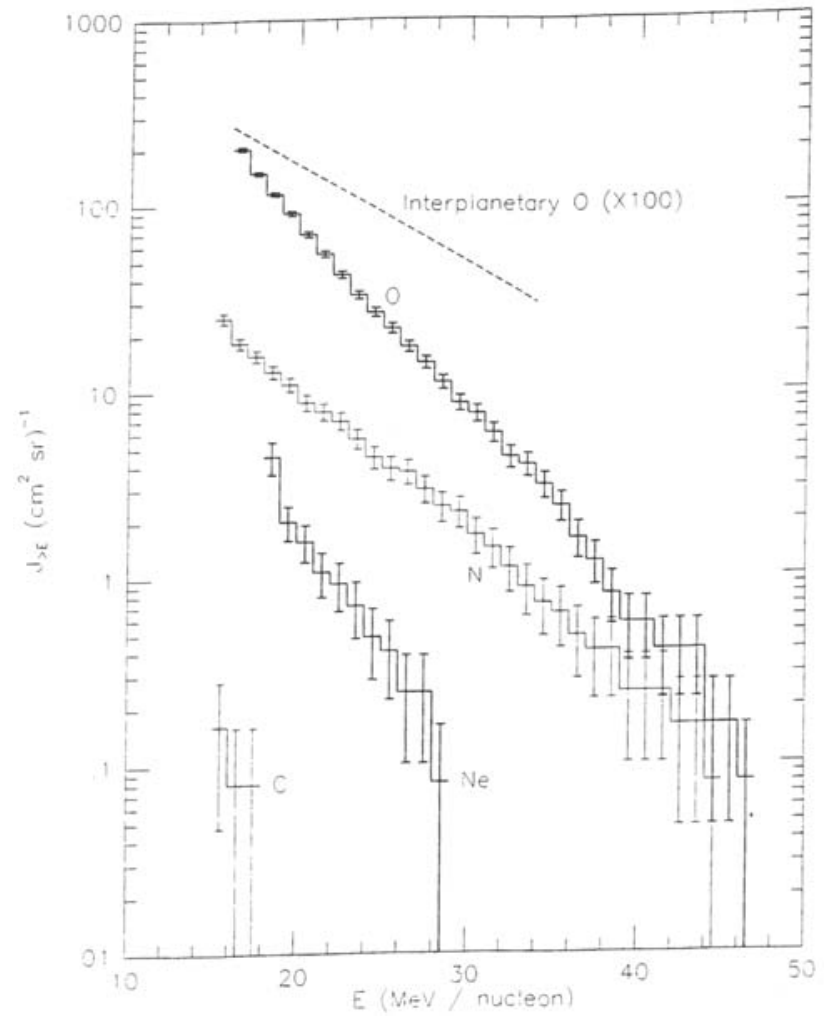

Figure 6. Integral energy spectra of trapped C, N, $\mathrm{O}$, and $\mathrm{Ne}$ tluences. The relative interplanetary $\mathrm{O}$ spectrum is also shown.

Cummings, J. R., A. C. Cummings, R. A. Mewaldt, R. S. Selesnick, E. C. Stone, and T. T. von Rosenvinge, New evidence for geomagnetically trapped anomalous cosmic rays, Geophys. Res. Lett., 20, 2003-2006, 1993.

Cummings, J. R., A. C. Cummings, R. A. Mewaldt, R. S. Selesnick, E. C. Stone, and T. T. von Rosenvinge, SAMPEX observations of geomagnetically trapped anomalous cosmic rays, in Proceedings of the 23rd Internatational Cosmic Ray Conference, Invited, Rapporteur and Highlight Papers, edited by D. A. Leahy, R. B. Hicks, and D. Venkatesan, pp. 475-482, World Scientific, Singapore, 1994.

Grigorov, N. L., M. A. Kondratyeva, M. I. Panasyuk, Ch. A. Tretyakova, J. H. Adams, Jr, J. B. Blake, M. Schulz, R. A. Mewaldt, and A. J. Tylka, Evidence for anomalous cosmic ray oxygen ions in the inner magnetosphere, Geophys. Res. Lett., 18, 1959-1962, 1991.

Mewaldt, R. A., A. C. Cummings, J. R. Cummings, E. C. Stone, B. Klecker, D. Hovestadt, M. Scholer, G. M. Mason, J. E. Mazur, D. C. Hamilton, T. T. von Rosenvinge, and J. B. Blake, The return of the anomalous component to $1 \mathrm{AU}$ in 1992, Geophys. Res. Lett., 20, 2263-2266, 1993.

Selesnick, R. S., A. C. Cummings, J. R. Cummings, R. A. Mewaldt, E. C. Stone, and T. T. von Rosenvinge, Geomagnetically trapped anomalous cosmic rays, J. Geophys. Res.in press, 1994.

R. S. Selesnick, J. R. Cummings, and R. A. Mewaldt, California Institute of Technology, mail code 220-47, Pasadena, CA 91125. 